\title{
Application of Ruscheweyh $q$-differential operator to analytic functions of reciprocal order
}

\author{
Shahid Mahmood, Saima Mustafa and Imran Khan
}

\begin{abstract}
The core object of this paper is to define and study new class of analytic function using Ruscheweyh $q$-differential operator. We also investigate a number of useful properties such as inclusion relation, coefficient estimates, subordination result,for this newly subclass of analytic functions.
\end{abstract}

Mathematics Subject Classification (2010): 30C45, 30C50.

Keywords: Analytic functions, Subordination, Functions with positive real part, Ruscheweyh $q$-differential operator, reciprocal order.

\section{Introduction}

Quantum calculus ( $q$-calculus) is simply the study of classical calculus without the notion of limits. The study of $q$-calculus attracted the researcher due to its applications in various branches of mathematics and physics, see detail [8]. Jackson [10, 12] was the first to give some application of $q$-calculus and introduced the $q$-analogue of derivative and integral. Later on Aral and Gupta $[5,6,7]$ defined the $q$-Baskakov Durrmeyer operator by using $q$-beta function while the author's in $[2,3,4]$ discussed the $q$-generalization of complex operators known as $q$-Picard and $q$-Gauss-Weierstrass singular integral operators. Recently, Kanas and Răducanu [13] defined $q$-analogue of Ruscheweyh differential operator using the concepts of convolution and then studied some of its properties. The application of this differential operator was further studied by Mohammed and Darus [1] and Mahmood and Sokół [14]. The aim of the current paper is to define a new class of analytic functions of reciprocal order involving $q$-differetial operator.

Let $\mathcal{A}$ be the class of functions having the form

$$
f(z)=z+\sum_{n=2}^{\infty} a_{n} z^{n}
$$


which are analytic in the open unit disk $\mathbb{U}=\{z \in \mathbb{C}:|z|<1\}$. Let $\mathcal{M}(\alpha)$ denote a subclass of $\mathcal{A}$ consisting of functions which satisfy the inequality

$$
\mathfrak{R e} \frac{z f^{\prime}(z)}{f(z)}<\alpha \quad(z \in \mathbb{U}),
$$

for some $\alpha(\alpha>1)$. And let $\mathcal{N}(\alpha)$ be the subclass of $\mathcal{A}$ consisting of functions $f$ which satisfy the inequality:

$$
\mathfrak{R e} \frac{\left(z f^{\prime}(z)\right)^{\prime}}{f^{\prime}(z)}<\alpha \quad(z \in \mathbb{U}),
$$

for some $\alpha(\alpha>1)$. These classes were studied by Owa et al. [16, 18]. Shams et al. [20] have introduced the $k$-uniformly starlike $\mathcal{S D}(k, \alpha)$ and $k$-uniformly convex $\mathcal{C D}(k, \alpha)$ of order $\alpha$, for some $k(k \geq 0)$ and $\alpha(0 \leq \alpha<1)$. Using these ideas in above defined classes, Junichi et al. [17] introduced the following classes.

Definition 1.1. Let $f \in \mathcal{A}$. Then $f$ is said to be in class $\mathcal{M D}(k, \alpha)$ if it satisfies

$$
\mathfrak{R e} \frac{z f^{\prime}(z)}{f(z)}<k\left|\frac{z f^{\prime}(z)}{f(z)}-1\right|+\alpha \quad(z \in \mathbb{U}),
$$

for some $\alpha(\alpha>1)$ and $k(k \leq 0)$.

Definition 1.2. An analytic function $f$ of the form (1.1) belongs to the class $\mathcal{N D}(k, \alpha)$, if and only if

$$
\mathfrak{R e} \frac{\left(z f^{\prime}(z)\right)^{\prime}}{f^{\prime}(z)}<k\left|\frac{\left(z f^{\prime}(z)\right)^{\prime}}{f^{\prime}(z)}-1\right|+\alpha \quad(z \in \mathbb{U}),
$$

for some $\alpha(\alpha>1)$ and $k(k \leq 0)$.

If $f$ and $g$ are analytic in $\mathbb{U}$, we say that $f$ is subordinate to $g$, written as $f \prec g$ or $f(z) \prec g(z)$, if there exists a Schwarz function $w$, which is analytic in $\mathbb{U}$ with $w(0)=0$ and $|w(z)|<1$ such that $f(z)=g(w(z))$. Furthermore, if the function $g(z)$ is univalent in $\mathbb{U}$, then we have the following equivalence holds, see $[11,15]$.

$$
f(z) \prec g(z) \quad(z \in \mathbb{U}) \Longleftrightarrow f(0)=g(0) \quad \text { and } \quad f(\mathbb{U}) \subset g(\mathbb{U}) .
$$

For two analytic functions

$$
f(z)=\sum_{n=1}^{\infty} a_{n} z^{n} \quad g(z)=\sum_{n=1}^{\infty} b_{n} z^{n} \quad(z \in \mathbb{U})
$$

For $t \in \mathbb{R}$ and $q>0, q \neq 1$, the number $[t, q]$ is defined in [14] as

$$
[t, q]=\frac{1-q^{t}}{1-q}, \quad[0, q]=0 .
$$

For any non-negative integer $n$ the $q$-number shift factorial is defined by

$$
[n, q] !=[1, q][2, q][3, q] \cdots[n, q], \quad([0, q] !=1) .
$$

We have $\lim _{q \rightarrow 1}[n, q]=n$. Throughout in this paper we will assume $q$ to be fixed number between 0 and 1 .

The $q$-derivative operator or $q$-difference operator for $f \in \mathcal{A}$ is defined as

$$
\partial_{q} f(z)=\frac{f(q z)-f(z)}{z(q-1)}, z \in \mathbb{U} .
$$


It can easily be seen that for $n \in \mathbb{N}:=\{1,2,3, \ldots\}$ and $z \in \mathbb{U}$

$$
\partial_{q} z^{n}=[n, q] z^{n-1}, \quad \partial_{q}\left\{\sum_{n=1}^{\infty} a_{n} z^{n}\right\}=\sum_{n=1}^{\infty}[n, q] a_{n} z^{n-1} .
$$

The $q$-generalized Pochhammer symbol for $t \in \mathbb{R}$ and $n \in \mathbb{N}$ is defined as

$$
[t, q]_{n}=[t, q][t+1, q][t+2, q] \cdots[t+n-1, q],
$$

and for $t>0$, let $q$-gamma function is defined as

$$
\Gamma_{q}(t+1)=[t, q] \Gamma_{q}(t) \text { and } \Gamma_{q}(1)=1 .
$$

Definition 1.3. [14] For a function $f(z) \in \mathcal{A}$, the Ruscheweyh $q$-differential operator is defined as

$$
\mathfrak{D}_{q}^{\mu} f(z)=\phi(q, \mu+1 ; z) * f(z)=z+\sum_{n=2}^{\infty} \Phi_{n-1} a_{n} z^{n}, \quad(z \in \mathbb{U} \text { and } \mu>-1),
$$

where

$$
\phi(q, \mu+1 ; z)=z+\sum_{n=2}^{\infty} \Phi_{n-1} z^{n}
$$

and

$$
\Phi_{n-1}=\frac{\Gamma_{q}(\mu+n)}{[n-1, q] ! \Gamma_{q}(\mu+1)}=\frac{[\mu+1, q]_{n-1}}{[n-1, q] !} .
$$

From (1.2), it can be seen that

$$
L_{q}^{0} f(z)=f(z) \text { and } L_{q}^{1} f(z)=z \partial_{q} f(z),
$$

and

$$
\begin{gathered}
L_{q}^{m} f(z)=\frac{z \partial_{q}^{m}\left(z^{m-1} f(z)\right)}{[m, q] !}, \quad(m \in \mathbb{N}) . \\
\lim _{q \rightarrow 1^{-}} \phi(q, \mu+1 ; z)=\frac{z}{(1-z)^{\mu+1}},
\end{gathered}
$$

and

$$
\lim _{q \rightarrow 1^{-}} \mathfrak{D}_{q}^{\mu} f(z)=f(z) * \frac{z}{(1-z)^{\mu+1}} .
$$

This shows that in case of $q \rightarrow 1^{-}$, the Ruscheweyh $q$-differential operator reduces to the Ruscheweyh differential operator $D^{\delta}(f(z))$ (see [19]). From (1.2) the following identity can easily be derived.

$$
z \partial \mathfrak{D}_{q}^{\mu} f(z)=\left(1+\frac{[\mu, q]}{q^{\mu}}\right) \mathfrak{D}_{q}^{\mu} f(z)-\frac{[\mu, q]}{q^{\mu}} \mathfrak{D}_{q}^{\mu} f(z) .
$$

If $q \rightarrow 1^{-}$, then

$$
z\left(\mathfrak{D}_{q}^{\mu} f(z)\right)^{\prime}=(1+\mu) \mathfrak{D}_{q}^{\mu} f(z)-\mu \mathfrak{D}_{q}^{\mu} f(z) .
$$

Now using the Ruscheweyh $q$-differential operator, we define the following class. 
Definition 1.4. Let $f \in \mathcal{A}$. Then $f$ is in the class $\mathcal{K} \mathcal{D}_{q}(k, \alpha, \gamma)$ if

$$
\mathfrak{R e}\left\{1+\frac{1}{\gamma}\left(\frac{z \partial_{q} \mathfrak{D}_{q}^{\mu} f(z)}{\mathfrak{D}_{q}^{\mu} f(z)}-1\right)\right\}<k\left|\frac{1}{\gamma}\left(\frac{z \partial_{q} \mathfrak{D}_{q}^{\mu} f(z)}{\mathfrak{D}_{q}^{\mu} f(z)}-1\right)\right|+\alpha,
$$

for some $k(k \leq 0), \alpha(\alpha>1)$ and for some $\gamma \in \mathbb{C} \backslash\{0\}$.

We note that $\mathcal{L D}_{2}^{0}(1,1, \alpha)=\mathcal{M}(\alpha)$ and $\mathcal{L D}_{1}^{0}(1,1, \alpha)=\mathcal{N}(\alpha)$, the classes introduced by Owa et al. [16, 18]. When we take $\gamma=1,2, c=1$, and $a=1$ the class $\mathcal{K} \mathcal{D}_{q}(k, \alpha, \gamma)$ reduces to the classes $\mathcal{M D}(k, \alpha)$ and $\mathcal{N D}(k, \alpha)$ (see [17]). For $1<\alpha<4 / 3$ the classes $\mathcal{M}(\alpha)$ and $\mathcal{N}(\alpha)$ were investigated by Uralegaddi et al. [21].

\section{Preliminary results}

Lemma 2.1. [9] For a positive integer $t$, we have

$$
\sigma \sum_{j=1}^{t} \frac{(\sigma)_{j-1}}{(j-1) !}=\frac{(\sigma)_{t}}{(t-1) !}
$$

Proof. Consider

$$
\begin{aligned}
& \sigma \sum_{j=1}^{t} \frac{(\sigma)_{j-1}}{(j-1) !} \\
= & \sigma\left(1+\frac{\sigma}{1}+\frac{(\sigma)_{2}}{2 !}+\frac{(\sigma)_{3}}{3 !}+\frac{(\sigma)_{4}}{4 !}+\cdots+\frac{(\sigma)_{t-1}}{(t-1) !}\right) \\
= & \sigma(1+\sigma)\left(1+\frac{\sigma}{2}+\frac{\sigma(\sigma+2)}{2 \times 3}+\cdots+\frac{\sigma(\sigma+2) \cdots(\sigma+t-2)}{2 \times \cdots \times(t-1)}\right) \\
= & \sigma(1+\sigma) \frac{(\sigma+2)}{2}\left(1+\frac{\sigma}{3}+\cdots+\frac{\sigma(\sigma+3) \cdots(\sigma+t-2)}{3 \times 4 \times \cdots \times(t-1)}\right) \\
= & \sigma(1+\sigma) \frac{(\sigma+2)}{2} \frac{(\sigma+3)}{3}\left(1+\frac{\sigma}{4}+\cdots+\frac{\sigma(\sigma+4) \cdots(\sigma+t-2)}{4 \times \cdots \times(t-1)}\right) \\
= & \sigma(1+\sigma) \frac{(\sigma+2)}{2} \frac{(\sigma+3)}{3} \frac{(\sigma+4)}{4}\left(1+\frac{\sigma}{5}+\cdots+\frac{\sigma \cdot \cdots+t}{5 \times 6 \times \cdots \times(t-1)}\right) \\
= & \sigma(1+\sigma) \frac{(\sigma+2)}{2} \frac{(\sigma+3)}{3} \frac{(\sigma+4)}{4} \cdots\left(1+\frac{\sigma}{t-1}\right) \\
= & \sigma(1+\sigma) \frac{(\sigma+2)}{2} \frac{(\sigma+3)}{3} \frac{(\sigma+4)}{4} \cdots\left(\frac{\sigma+(t-1)}{t-1}\right) \\
= & \frac{(\sigma)_{t}}{(t-1) !} \cdot
\end{aligned}
$$




\section{Main results}

With the help of the definition of $\mathcal{K D}_{q}(k, \alpha, \gamma)$, we prove the following results.

Theorem 3.1. If $f(z) \in \mathcal{K} \mathcal{D}_{q}(k, \alpha, \gamma)$, then

$$
f(z) \in \mathcal{K D}_{q}\left(0, \frac{\alpha-k}{1-k}, \gamma\right) \text {. }
$$

Proof. Because $k \leq 0$, we have

$$
\begin{aligned}
\mathfrak{R e}\left\{1+\frac{1}{\gamma}\left(\frac{z \partial_{q} \mathfrak{D}_{q}^{\mu} f(z)}{\mathfrak{D}_{q}^{\mu} f(z)}-1\right)\right\} & <k\left|\frac{1}{\gamma}\left(\frac{z \partial_{q} \mathfrak{D}_{q}^{\mu} f(z)}{\mathfrak{D}_{q}^{\mu} f(z)}-1\right)\right|+\alpha, \\
& \leq k \mathfrak{R e}\left(\frac{1}{\gamma}\left(\frac{z \partial_{q} \mathfrak{D}_{q}^{\mu} f(z)}{\mathfrak{D}_{q}^{\mu} f(z)}-1\right)\right)+\alpha-k,
\end{aligned}
$$

which implies that

$$
(1-k) \mathfrak{R e} \frac{1}{\gamma}\left(\frac{z \partial_{q} \mathfrak{D}_{q}^{\mu} f(z)}{\mathfrak{D}_{q}^{\mu} f(z)}-1\right)<\alpha-k .
$$

After simplification, we obtain

$$
\mathfrak{R e}\left[1+\frac{1}{\gamma}\left(\frac{z \partial_{q} \mathfrak{D}_{q}^{\mu} f(z)}{\mathfrak{D}_{q}^{\mu} f(z)}-1\right)\right]<\frac{\alpha-k}{1-k},(k \leq 0, \alpha>1 \text { and }) .
$$

This completes the proof.

Theorem 3.2. If $f(z) \in \mathcal{K} \mathcal{D}_{q}(k, \alpha, \gamma)$ and if $f(z)$ has the form (1.1), then

$$
\left|a_{n}\right| \leq \frac{(\sigma)_{n-1}}{(n-1) ! \Phi_{n-1}}
$$

where

$$
\sigma=\frac{2|\gamma|(\alpha-1)}{q(1-k)}
$$

Proof. Let us define a function

$$
p(z)=\frac{(\alpha-k)-(1-k)\left[1+\frac{1}{\gamma}\left(\frac{z \partial_{q} \mathfrak{D}_{q}^{\mu} f(z)}{\mathfrak{D}_{q}^{\mu} f(z)}-1\right)\right]}{\alpha-1} .
$$

Then $p(z)$ is analytic in $\mathbb{U}, p(0)=1$ and $\mathfrak{R e}\{p(z)\}>0$ for $z \in \mathbb{U}$. We can write

$$
\left[1+\frac{1}{\gamma}\left(\frac{z \partial_{q} \mathfrak{D}_{q}^{\mu} f(z)}{\mathfrak{D}_{q}^{\mu} f(z)}-1\right)\right]=\frac{(\alpha-k)-(\alpha-1) p(z)}{1-k}
$$

If we take $p(z)=1+\sum_{n=1}^{\infty} p_{n} z^{n}$, then $(3.5)$ can be written as

$$
z \partial_{q} \mathfrak{D}_{q}^{\mu} f(z)-\mathfrak{D}_{q}^{\mu} f(z)=-\frac{\gamma(\alpha-1)}{1-k}\left(\mathfrak{D}_{q}^{\mu} f(z)\right)\left(\sum_{n=1}^{\infty} p_{n} z^{n}\right) .
$$

this implies that

$$
\left[\sum_{n=2}^{\infty} q[n-1] \Phi_{n-1} a_{n} z^{n}\right]=-\frac{\gamma(\alpha-1)}{1-k}\left(\sum_{n=1}^{\infty} \Phi_{n-1} a_{n} z^{n}\right)\left(\sum_{n=1}^{\infty} p_{n} z^{n}\right) .
$$


Using Cauchy product $\left(\sum_{n=1}^{\infty} x_{n}\right) \cdot\left(\sum_{n=1}^{\infty} y_{n}\right)=\sum_{j=1}^{\infty} \sum_{k=1}^{j} x_{k} y_{k-j}$, we obtain

$$
q[n-1] \Phi_{n-1} a_{n} z^{n}=-\frac{\gamma(\alpha-1)}{1-k} \sum_{n=2}^{\infty}\left(\sum_{j=1}^{n-1} \Phi_{j-1} a_{j} p_{n-j}\right) z^{n}
$$

Comparing the coefficients of $n t h$ term on both sides, we obtain

$$
a_{n}=\frac{-\gamma(\alpha-1)}{q[n-1] \Phi_{n-1}(1-k)} \sum_{j=1}^{n-1} \Phi_{j-1} a_{j} p_{n-j} .
$$

By taking absolute value and applying triangle inequality, we get

$$
\left|a_{n}\right| \leq \frac{|\gamma|(\alpha-1)}{q[n-1] \Phi_{n-1}(1-k)} \sum_{j=1}^{n-1} \Phi_{j-1}\left|a_{j}\right|\left|p_{n-j}\right| .
$$

Applying the coefficient estimates $\left|p_{n}\right| \leq 2(n \geq 1)$ for Caratheodory functions [11], we obtain

$$
\begin{aligned}
\left|a_{n}\right| & \leq \frac{2|\gamma|(\alpha-1)}{q[n-1] \Phi_{n-1}(1-k)} \sum_{j=1}^{n-1} \Phi_{j-1}\left|a_{j}\right| \\
& =\frac{\sigma}{[n-1] \Phi_{n-1}} \sum_{j=1}^{n-1} \psi_{j-1}\left|a_{j}\right|,
\end{aligned}
$$

where $\sigma=2|\gamma|(\alpha-1) / q(1-k)$. To prove (3.2) we apply mathematical induction. So for $n=2$, we have from (3.6)

$$
\left|a_{2}\right| \leq \frac{\sigma}{\Phi_{1}}=\frac{(\sigma)_{2-1}}{[2-1] ! \Phi_{2-1}}
$$

which shows that (3.2) holds for $n=2$. For $n=3$, we have from (3.6)

$$
\left|a_{3}\right| \leq \frac{\sigma}{[3-1] \Phi_{3-1}}\left\{1+\Phi_{1}\left|a_{2}\right|\right\}
$$

using (3.7), we have

$$
\left|a_{3}\right| \leq \frac{\sigma}{[2] \Phi_{2}}(1+\sigma)=\frac{(\sigma)_{3-1}}{[3-1] \Phi_{3-1}},
$$

which shows that (3.2) holds for $n=3$. Let us assume that (3.2) is true for $n \leq t$, that is,

$$
\left|a_{t}\right| \leq \frac{(\sigma)_{t-1}}{[t-1] ! \Phi_{t-1}} \quad j=1,2, \ldots, t
$$


Using (3.6) and (3.8), we have

$$
\begin{aligned}
\left|a_{t+1}\right| & \leq \frac{\sigma}{t \Phi_{t}} \sum_{j=1}^{t} \Phi_{j-1}\left|a_{j}\right| \\
& \leq \frac{\sigma}{t \Phi_{t}} \sum_{j=1}^{t} \psi_{j-1} \frac{(\sigma)_{j-1}}{[j-1] ! \Phi_{j-1}} \\
& =\frac{\sigma}{t \Phi_{t}} \sum_{j=1}^{t} \frac{(\sigma)_{j-1}}{[j-1] !}
\end{aligned}
$$

Applying (2.1), we have

$$
\begin{aligned}
\left|a_{t+1}\right| & \leq \frac{1}{t \Phi_{t}} \frac{(\sigma)_{t}}{[t-1] !} \\
& =\frac{1}{\Phi_{t}} \frac{(\sigma)_{t}}{[t] !}
\end{aligned}
$$

Consequently, using mathematical induction, we have proved that (3.2) holds true for all $n, n \geq 2$. This completes the proof.

Theorem 3.3. If a function $f \in \mathcal{K} \mathcal{D}_{q}(k, \alpha, \gamma)$, then

$$
\begin{gathered}
\frac{z \partial_{q} \mathfrak{D}_{q}^{\mu} f(z)}{\mathfrak{D}_{q}^{\mu} f(z)} \prec 1+2\left(\alpha_{1}-1\right)-\frac{2\left(\alpha_{1}-1\right)}{1-z}(z \in \mathbb{U}), \\
\alpha_{1}=\frac{\alpha-k}{1-k} .
\end{gathered}
$$

Proof. If $f(z) \in \mathcal{K} \mathcal{D}_{q}(k, \alpha, \gamma)$, then by (3.1)

$$
\mathfrak{R e}\left\{1+\frac{1}{\gamma}\left(\frac{z \partial_{q} \mathfrak{D}_{q}^{\mu} f(z)}{\mathfrak{D}_{q}^{\mu} f(z)}-1\right)\right\}<\alpha_{1} .
$$

Then there exists a Schwarz function $w(z)$ such that

$$
\frac{\alpha_{1}-\left\{1+\frac{1}{\gamma}\left(\frac{z \partial_{q} \mathfrak{D}_{q}^{\mu} f(z)}{\mathfrak{D}_{q}^{\mu} f(z)}-1\right)\right\}}{\alpha_{1}-1}=\frac{1+w(z)}{1-w(z)},
$$

and

$$
\mathfrak{R e}\left\{\frac{1+w(z)}{1-w(z)}\right\}>0, \quad(z \in \mathbb{U}) .
$$

Therefore, from (3.12), we obtain

$$
\frac{z \partial_{q} \mathfrak{D}_{q}^{\mu} f(z)}{\mathfrak{D}_{q}^{\mu} f(z)}=1+\gamma\left(\alpha_{1}-1\right)\left(1-\frac{1+w(z)}{1-w(z)}\right) .
$$

This gives

$$
\frac{z \partial_{q} \mathfrak{D}_{q}^{\mu} f(z)}{\mathfrak{D}_{q}^{\mu} f(z)}=1+2 \gamma\left(\alpha_{1}-1\right)-\frac{2 \gamma\left(\alpha_{1}-1\right)}{1-w(z)}
$$


and hence

$$
\frac{z \partial_{q} \mathfrak{D}_{q}^{\mu} f(z)}{\mathfrak{D}_{q}^{\mu} f(z)} \prec 1+2 \gamma\left(\alpha_{1}-1\right)-\frac{2 \gamma\left(\alpha_{1}-1\right)}{1-z}(z \in \mathbb{U}) .
$$

which was required in (3.9).

Theorem 3.4. If function $f \in \mathcal{K} \mathcal{D}_{q}(k, \alpha, \gamma)$, then we have

$$
\frac{1-\left[1+2 \gamma\left(\alpha_{1}-1\right)\right] r}{1-r} \leq \mathfrak{R e}\left\{\frac{z \partial_{q} \mathfrak{D}_{q}^{\mu} f(z)}{\mathfrak{D}_{q}^{\mu} f(z)}\right\} \leq \frac{1+\left[1+2 \gamma\left(\alpha_{1}-1\right)\right] r}{1+r},
$$

for $|z|=r<1$ and $\alpha_{1}$ is defined by (3.10).

Proof. By the virtue of Theorem (3.3), let us take the function $\phi(z)$ defined by

$$
\phi(z)=1+2 \gamma\left(\alpha_{1}-1\right)-\frac{2 \gamma\left(\alpha_{1}-1\right)}{1-z} \quad(z \in \mathbb{U}) .
$$

Letting $z=r e^{i \theta}(0 \leq r<1)$, we see that

$$
\mathfrak{R e} \phi(z)=1+2 \gamma\left(\alpha_{1}-1\right)+\frac{2 \gamma\left(1-\alpha_{1}\right)(1-r \cos \theta)}{1+r^{2}-2 r \cos \theta} .
$$

Let us define

$$
\psi(t)=\frac{1-r t}{1+r^{2}-2 r t} \quad(t=\cos \theta)
$$

Since $\psi^{\prime}(t)=\frac{r\left(1-r^{2}\right)}{\left(1+r^{2}-2 r t\right)^{2}} \geq 0$, because $r<1$. Therefore we get

$$
1+2 \gamma\left(\alpha_{1}-1\right)-\frac{2 \gamma\left(\alpha_{1}-1\right)}{1-r} \leq \mathfrak{R e} \phi(z) \leq 1+2 \gamma\left(\alpha_{1}-1\right)-\frac{2 \gamma\left(\alpha_{1}-1\right)}{1+r}
$$

After simplification, we have

$$
\frac{1-\left[1+2 \gamma\left(\alpha_{1}-1\right)\right] r}{1-r} \leq \mathfrak{R e} \phi(z) \leq \frac{\left.1+\left[1+2 \gamma\left(\alpha_{1}-1\right)\right)\right] r}{1+r} .
$$

Since we note that $\frac{z \partial_{q} \mathfrak{D}_{q}^{\mu} f(z)}{\mathfrak{D}_{q}^{\mu} f(z)} \prec \phi(z),(z \in \mathbb{U})$ by Theorem 3.3 and $\phi(z)$ is analytic in $\mathbb{U}$, we proved the inequality (3.13).

Theorem 3.5. If $f \in \mathcal{A}$ satisfies

$$
\left|\frac{z \partial_{q} \mathfrak{D}_{q}^{\mu} f(z)}{\mathfrak{D}_{q}^{\mu} f(z)}-1\right|<\frac{(\alpha-1)|\gamma|}{(1-k)} \quad z \in \mathbb{U},
$$

for some $k(k \leq 0), \alpha(\alpha>1)$ and $\gamma \in \mathbb{C} \backslash\{0\}$. Then $f \in \mathcal{K} \mathcal{D}_{q}(k, \alpha, \gamma)$. 
Proof.

$$
\begin{aligned}
& \left|\frac{z \partial_{q} \mathfrak{D}_{q}^{\mu} f(z)}{\mathfrak{D}_{q}^{\mu} f(z)}-1\right|<\frac{(\alpha-1)|\gamma|}{(1-k)} \\
\Rightarrow & \left|\frac{1}{\gamma}\left(\frac{z \partial_{q} \mathfrak{D}_{q}^{\mu} f(z)}{\mathfrak{D}_{q}^{\mu} f(z)}-1\right)\right|<\frac{\alpha-1}{1-k} \\
\Rightarrow & (1-k)\left|\frac{1}{\gamma}\left(\frac{z \partial_{q} \mathfrak{D}_{q}^{\mu} f(z)}{\mathfrak{D}_{q}^{\mu} f(z)}-1\right)\right|+1<\alpha \\
\Rightarrow & \left|\frac{1}{\gamma}\left(\frac{z \partial_{q} \mathfrak{D}_{q}^{\mu} f(z)}{\mathfrak{D}_{q}^{\mu} f(z)}-1\right)\right|+1<k\left|\frac{1}{\gamma}\left(\frac{z \partial_{q} \mathfrak{D}_{q}^{\mu} f(z)}{\mathfrak{D}_{q}^{\mu} f(z)}-1\right)\right|+\alpha \\
\Rightarrow & \mathfrak{R e}\left\{1+\frac{1}{\gamma}\left(\frac{z \partial_{q} \mathfrak{D}_{q}^{\mu} f(z)}{\mathfrak{D}_{q}^{\mu} f(z)}-1\right)\right\}+1<k\left|\frac{1}{\gamma}\left(\frac{z \partial_{q} \mathfrak{D}_{q}^{\mu} f(z)}{\mathfrak{D}_{q}^{\mu} f(z)}-1\right)\right|+\alpha \\
\Rightarrow & f \in \mathcal{L} \mathcal{D}_{b}^{k}(a, c, \beta)
\end{aligned}
$$

Corollary 3.6. Let $f \in \mathcal{A}$ be of the form (1.1) and satisfies

$$
\left|\frac{\sum_{n=2}^{\infty}[n-1] \Phi_{n-1} a_{n} z^{n-1}}{1+\sum_{n=2}^{\infty} \Phi_{n-1} a_{n} z^{n-1}}\right|<\frac{(\alpha-1)|\gamma|}{q(1-k)} \quad z \in \mathbb{U},
$$

for some $k(k \leq 0), \beta(\beta>1)$ and for some $b \in \mathbb{C} \backslash\{0\}$. Then $f \in \mathcal{K D}_{q}(k, \alpha, \gamma)$..

Proof. We have

$$
\mathfrak{D}_{q}^{\mu} f(z)=z+\sum_{n=2}^{\infty} \Phi_{n-1} a_{n} z^{n}
$$

and by (1.5)

$$
z \partial \mathfrak{D}_{q}^{\mu} f(z)=z+\sum_{n=2}^{\infty}[n] \Phi_{n-1} a_{n} z^{n} .
$$

Therefore, (3.14) follows immediately (3.15).

Theorem 3.7. Let $f \in \mathcal{A}$ be of the form (1.1) and satisfies

$$
\sum_{n=2}^{\infty}([n-1]+y)\left|\Phi_{n-1}\right|\left|a_{n}\right|<y \quad z \in \mathbb{U}
$$

for some $k(k \leq 0), \beta(\beta>1)$ and for some $b \in \mathbb{C} \backslash\{0\}$ and where

$$
y=\frac{(\alpha-1)|\gamma|}{q(1-k)}>0 \text {. }
$$

Then $f \in \mathcal{K D}_{q}(k, \alpha, \gamma)$. 
Proof. We have

$$
\begin{aligned}
& \sum_{n=2}^{\infty}([n-1]+y)\left|\Phi_{n-1}\right|\left|a_{n}\right|<y \\
\Rightarrow & \sum_{n=2}^{\infty}([n-1]+y)\left|\Phi_{n-1}\right|\left|a_{n}\right|<y-y \sum_{n=2}^{\infty}\left|\Phi_{n-1}\right|\left|a_{n}\right| \\
\Rightarrow & 0<y-y \sum_{n=2}^{\infty}\left|\Phi_{n-1}\right|\left|a_{n}\right| \\
\Rightarrow & 0<y-y \sum_{n=2}^{\infty}\left|\Phi_{n-1}\right|\left|a_{n}\right|\left|z^{n-1}\right| \\
\Rightarrow & 0<y\left|1+\sum_{n=2}^{\infty} \Phi_{n-1} a_{n} z^{n-1}\right|
\end{aligned}
$$

We have

$$
\begin{aligned}
& \sum_{n=2}^{\infty}([n-1]+y)\left|\Phi_{n-1}\right|\left|a_{n}\right|<y \\
\Rightarrow & \sum_{n=2}^{\infty}([n-1]+y)\left|\Phi_{n-1}\right|\left|a_{n}\right|\left|z^{n-1}\right|<y \\
\Rightarrow & \sum_{n=2}^{\infty}[n-1]\left|\Phi_{n-1}\right|\left|a_{n}\right|\left|z^{n-1}\right|<y-y \sum_{n=2}^{\infty}\left|\Phi_{n-1}\right|\left|a_{n}\right|\left|z^{n-1}\right| \\
\Rightarrow & \left|\sum_{n=2}^{\infty}[n-1] \Phi_{n-1} a_{n} z^{n-1}\right|<y\left|1+\sum_{n=2}^{\infty} \Phi_{n-1} a_{n} z^{n-1}\right| \\
\Rightarrow & \left|\frac{\sum_{n=2}^{\infty}[n-1] \Phi_{n-1} a_{n} z^{n-1}}{1+\sum_{n=2}^{\infty} \Phi_{n-1} a_{n} z^{n-1}}\right|<y,
\end{aligned}
$$

because of (3.17). By (3.15) it follows $f \in \mathcal{L D}_{b}^{k}(a, c, \beta)$.

\section{References}

[1] Aldweby, H., Darus, M., Some subordination results on q-analogue of Ruscheweyh differential operator, Abstr. Appl. Anal., Vol. 2014, Art. ID 958563, 6 pages.

[2] Anastassiu, G.A., Gal, S.G., Geometric and approximation properties of some singular integrals in the unit disk, J. Inequal. Appl., Vol. 2006, Art. ID 17231, 19 pages.

[3] Anastassiu, G.A., Gal, S.G., Geometric and approximation properties of generalized singular integrals, J. Korean Math. Soc., 23(2006), no. 2, 425-443.

[4] Aral, A., On the generalized Picard and Gauss Weierstrass singular integrals, J. Comput. Anal. Appl., 8(2006), no. 3, 249-261.

[5] Aral, A., Gupta, V., On q-Baskakov type operators, Demonstr. Math., 42(2009), no. 1, 109-122. 
[6] Aral, A., Gupta, V., On the Durrmeyer type modification of the q-Baskakov type operators, Nonlinear Anal. Theory, Methods and Appl., 72(2010), no. 3-4, 1171-1180.

[7] Aral, A., Gupta, V., Generalized q-Baskakov operators, Math. Slovaca, 61(2011), no. 4, 619-634.

[8] Aral, A., Gupta, V., Agarwal, R.P., Applications of q-Calculus in Operator Theory, Springer, New York, NY, USA, 2013.

[9] Arif, M., Mahmood, S., Sokół, J., Dziok, J., New subclass of analytic functions in conical domain associated with a linear operator, Acta Math. Sci., 36B(2016), no. 3, 1-13.

[10] Jackson, F.H., On q-functions and a certain difference operator, Trans. Roy. Soc. Edinburgh, 46(1909), no. 2, 253-281.

[11] Goodman, A.W., Univalent Functions, Vol. I, II, Polygonal Publishing House, Washington, New Jersey, 1983.

[12] Jackson, F.H., On q-definite integrals, Quart. J. Pure Appl. Math., 41(1910), 193-203.

[13] Kanas, S., Raducanu, D., Some class of analytic functions related to conic domains, Math. Slovaca, 64(2014), no. 5, 1183-1196.

[14] Mahmmod, S., Sokół, J., New subclass of analytic functions in conical domain associated with Ruscheweyh q-differential operator, J. Results Math., 71(2017), 1345-1357.

[15] Miller, S.S., Mocanu, P.T., Differential Subordinations Theory and Applications, Marcel Decker Inc., New York, 2000.

[16] Nishiwaki, J., Owa, S., Coefficient estimates for certain classes of analytic functions, J. Inequal. Pure Appl. Math., 3(2002), 1-5.

[17] Nishiwaki, J., Owa, S., Certain classes of analytic functions concerned with uniformly starlike and convex functions, Appl. Math. Comput., 187(2007), 350-355.

[18] Owa, S., Srivastava, H.M., Some generalized convolution properties associated with cerain subclasses of analytic functions, J. Inequal. Pure Appl. Math., 3(2002), no. 3, 1-13.

[19] Ruscheweyh,St., New criteria for univalent functions, Proc. Amer. Math. Soc., 49(1975), 109-115.

[20] Shams, S., Kulkarni, S.R., Jahangiri, J.M., Classes of uniformly starlike and convex functions, Int. J. Math. Math. Sci., 55(2004), 2959-2961.

[21] Uralegaddi, B.A., Ganigi, M.D., Sarangi, S.M., Univalent functions with positive coefficients, Tamkang J. Math., 25(1994), 225-230.

Shahid Mahmood

Corresponding author

Department of Mechanical Engineering, Sarhad University of Science

and

I.T. Landi Akhun Ahmad, Hayatabad Link. Ring Road, Peshawar, Pakistan

e-mail: shahidmahmood757@gmail.com

Saima Mustafa

Department of Statistics \& Mathematics

PMAS-Arid Agriculture University, Rawalpindi

e-mail: saimamustafa28@gmail.com 
Imran Khan

Department of Basic Sciences and Islamyat University of Engineering and Technology Peshawar, Pakistan

e-mail: ikhanqau1@gmail.com 